\title{
An Extensible Framework for Web Sites Integration
}

\author{
Nadira Lammari ${ }^{1}$, Isabelle Comyn-Wattiau ${ }^{2}$ and Jacky Akoka ${ }^{3}$ \\ 1 Laboratoire CEDRIC-CNAM, 292 Rue Saint Martin, F-75141 Paris Cedex 03, \\ lammari@cnam.fr \\ 2 Université de Cergy-Pontoise et ESSEC, 2 Rue Adolphe Chauvin, F-95302 \\ Pontoise Cedex \\ 3 Laboratoire CEDRIC-CNAM et Institut National des Télécoms
}

\begin{abstract}
One possible way to build rapidly a web site for an organization or one of its business units is to collect existing web sites describing parts of the organization and to integrate them into a global unified web site. It allows the designer to reuse past developing efforts in order to produce a web site containing at least the union of the existing web sites. The main problem is to define a well-structured integrated web site. By analogy with view integration techniques developed in the past, this paper describes a method to perform such an integration process. Following the development of our approach, we analyze a real-life web sites integration and provide some insights on useful aspects of web site integration.
\end{abstract}

Key words: web site conceptual modelling, web site integration, view integration

\section{INTRODUCTION}

New information technologies bring new challenges. Companies have to cope with new business drivers as globalization, mergers, acquisitions, and process reengineering. To do so, companies exploit Internet, build e-commerce applications and develop new and 
improved web sites. E-commerce applications require a robust information technology infrastructure and the ability to rapidly design, develop and implement new web sites. In e-commerce era, time is the driver and information is the source of innovation. Being the first to market new ideas or to propagate a new image after a reorganization process or a merger is seen to be crucial. How can a company face changing environment and changing strategic plans ? One important way is by adapting existing web sites and e-commerce applications. Reducing the elapse time of any stage of the development life cycle is a way to become more proactive.

The integration of existing web sites, after a reorganization process within the business units of a company or after a merger or an acquisition have taken place, is a way to become more responsive to fast changing markets. The aim of this paper is precisely to present and illustrate the integration process of existing web sites. As for data or for schema integration, the web site integration process consists of four steps: (i) pre-integration, (ii) comparison, (iii) merging, and (iv) restructuring. In this paper, we will concentrate on the critical phases of comparison and merging. Our approach emphasizes the development of fast web sites integration, using specific algorithms based on an Entity-Relationship conceptual schema representing each existing web site. Similarities between the web pages and their structures is the basis for the merging step.

The paper is organized as follows. Section 2 describes the approach applied to perform web sites integration. Section 3 highlights the integration mechanism and its application to a case study. Section 4 is devoted to a presentation of the related literature. We conclude in Section 5 and present some areas of future research.

\section{THE INTEGRATION APPROACH - STEP BY STEP}

Our approach is based on the classical integration schema process composed of four main steps [3]:

- a pre-integration step which aims at transforming the initial schemas using a common target conceptual model,

- a comparison step which leads to the identification of correspondences between concepts belonging to the two schemas, 
- a merging step which builds the resulting schema from the two source schemas,

- finally, a restructuring or enrichment step to improve the quality of the integrated schema.

This process must be adapted to deal with web site integration (Figure 1). This is precisely the aim of this paper.

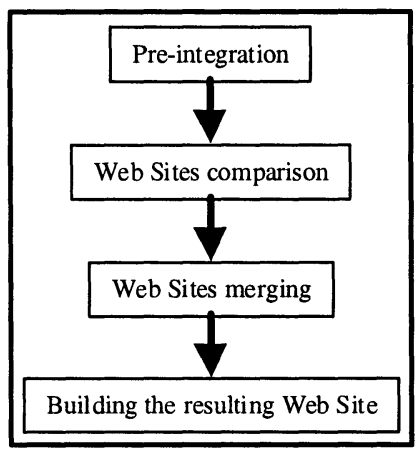

Figure 1. The web site integration process

\subsection{Pre-integration step}

The pre-integration step aims at representing the two web sites according to a common formalism. This common representation is necessary to allow the integrator - human or tool - to compare the web site contents. We have defined an Entity-Relationships conceptual schema whose instances are web site contents (Figure 2). This conceptual schema although simple is relevant for the integration process. It can be extended to include other aspects for an extended integration process.

Layout or aesthetic aspects of the sites are not represented since we perform a semantic web sites integration.

In this conceptual schema, a web site is composed of pages. A page contains links, either intra-site or extra-site links. Intra-site links may be intra-page or inter-page links. A page generally contains different text parts, and especially a set of titles structured at different levels. It contains also images, forms, lists and tables. In this paper, we consider titles, images, free texts, tables, lists, and forms. Other objects, such as 
sound, video, comments, frames and scripts will be considered in future papers.

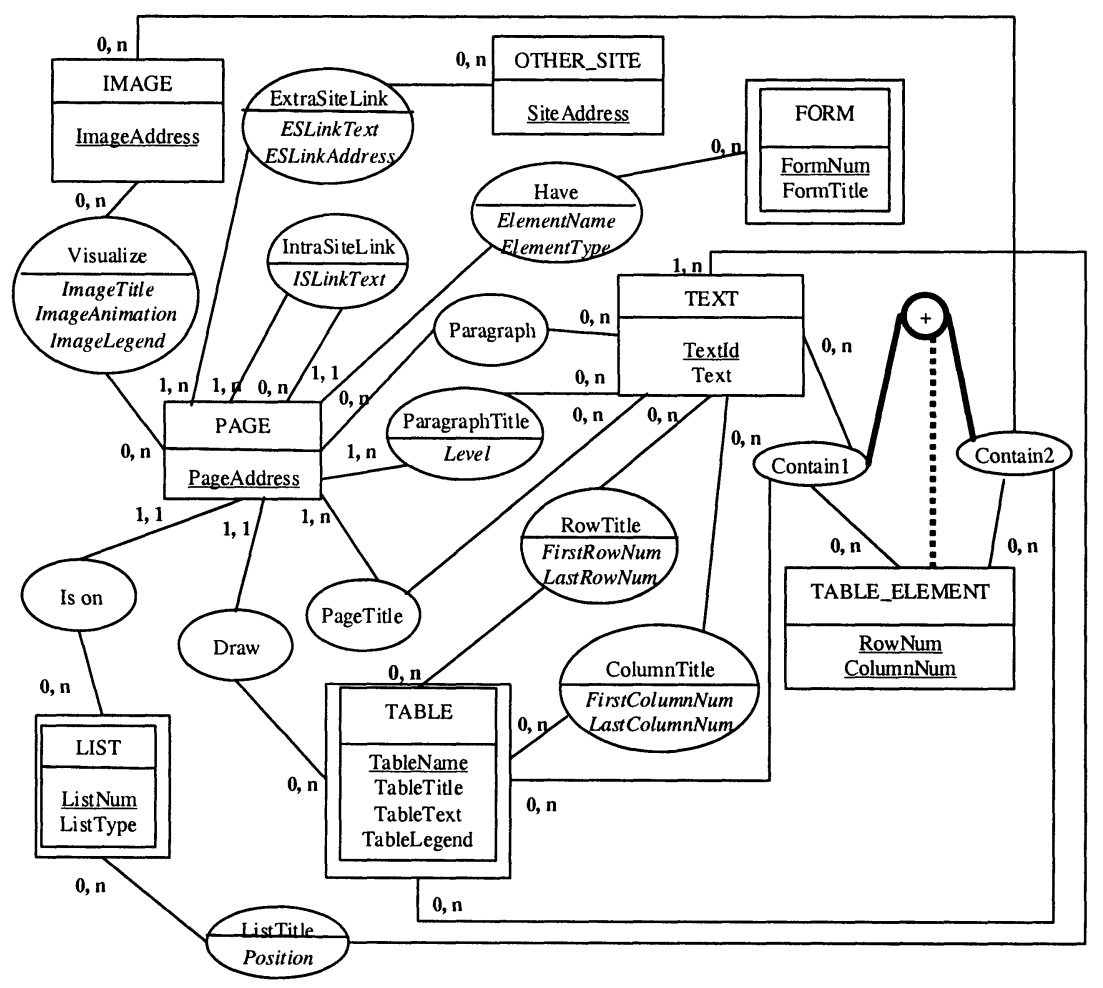

Figure 2. The web site conceptual model

The pre-integration step is composed of the three following phases:

- The purge of page descriptions. During this phase, the information not relevant to the integration process is removed from the page descriptions.

- The normalization phase consists in transforming the pages containing intra-link pages. The integration process is facilitated when web sites are in a canonical form without any intra-page link. At the end of this step, the web sites to be integrated define two instances of the conceptual schema described above. From each instance is constructed a graph whose nodes are web pages or smaller components and edges are hypertext links. This graph will be used 
during the merging step to perform the construction of the resulting web site.

\subsection{Comparison step}

The comparison step is conducted by an algorithm which is based on a navigation through the web site graphs. It starts with the two top nodes, i.e. the two nodes representing the two site welcome pages. At each step of the algorithm, the comparison of two web pages is performed. This comparison is guided by a matrix of comparable pages. We first present below the matrix structure and its generation. Secondly, we describe the comparison of pages.

\subsubsection{Generation of the matrix of comparable pages}

The comparison step is the bottle-neck of integration processes. This is due to the fact that it requires, in the worst case, a two-by-two comparison of all elements of both structures to be integrated. Therefore, it is crucial to define heuristics leading to an important decrease of the number of comparisons. Pursuing this objective, we build a matrix defining, for each couple of pages belonging to the two sites, whether these pages are to be compared.

In [12], we proposed an algorithm aiming at extracting from a set of relational tuples, the different entity-types embedded in the relation. This process allowed us to deduce entities and generalization links between them. In this paper, we adapt this algorithm to build classes of comparable web pages:

Let $\mathrm{n}$ be the number of structures taken into account by the integration process (for example, $n=4$ if we consider texts, titles, images and forms). Let Page1 be the set of the first web site pages and Page 2 the set of the second web site pages. Let $\mathrm{F}$ be a function defined as follows:

$$
\text { F: Page1 } \cup \text { Page } 2 \rightarrow \underbrace{\{0,1\} \times\{0,1\} \times \ldots \ldots \times\{0,1\}}_{\text {ntimes }}
$$


$\mathrm{P} \quad \mapsto \quad(\mathrm{v} 1, \mathrm{v} 2, \ldots, \mathrm{vn})$

where $\mathrm{vk}=0$ if the page $\mathrm{P}$ does not contain any structure of $\mathrm{k}$ type

$\mathrm{vk}=1$ otherwise.

The image of Page $1 \cup$ Page 2 using $F$ is a subset of $\{0,1\} \times\{0,1\} \times$ $\ldots \times\{0,1\}$

Let $R$ be the relation whose $n$ domains are $\{0,1\}$ representing the $n$ structure types taken into account.

The algorithm sketched above and described in [12] is performed on $R$ leading to an ISA hierarchy. In this hierarchy, an entity puts together web pages having at least one common structure type. If two web pages share several structure types, they will be present together in several entities and submitted to several comparisons. Notice that, in a same entity, web pages may belong to the same site. Obviously the comparison process will only compare pages grouped in a same entity but extracted from the two different sites.

The result of this phase is a two-dimension matrix C. A component $\mathrm{Ci}, \mathrm{j}$ contains the set of structures shared by the $\mathrm{i}$-th page of the first site and the $\mathrm{j}$-th page of the second site. If this set is empty, the pages are not compared. Their similarity is null.

\subsubsection{Similarity derivation}

The comparison step aims at defining, for each pair of comparable pages, a degree of similarity. This similarity is deduced from the aggregation of similarities between common type structures contained in the two pages. Therefore, we describe first the elementary similarities, measuring the common features of two structures, for example two texts or two images. Secondly, we propose an aggregation of these similarities in order to attain a decision for each pair of web pages by answering the following query: do these two web pages share enough common features to be considered as equivalent and merged into a unique one ?

The comparison of structures is based on several criteria. The criteria are different for each structure type. They are defined at 
different detail levels. For example, the comparison of two pages starts with the comparison of their paragraph titles. If these sets are found semantically similar, we compare their hierarchical levels- a sub-title level 3 in the first site is compared to all the second site subtitles. If both paragraph title sets are identical and paragraph title levels are identical, we compare the titles of the pages. If paragraph title sets, paragraph title levels and title pages are two by two identical, then the two pages will be considered as equivalent for this title criteria. The similarity is, in this case, equal to $100 \%$. On the contrary, two pages having no common title paragraph set and no common title page will be considered as dissimilar. The similarity is then equal to $0 \%$.

The similarity degree between two web pages according to each structure type is established by a rule set described in a decision table. Notice that these numerical values aim only at defining a total order between similarity cases. Table A tackles with Text structure type. . Table B describes similarities between list structures. Table $\mathrm{Cl}$ and $\mathrm{C} 2$ contains the rule set for the structure type Title

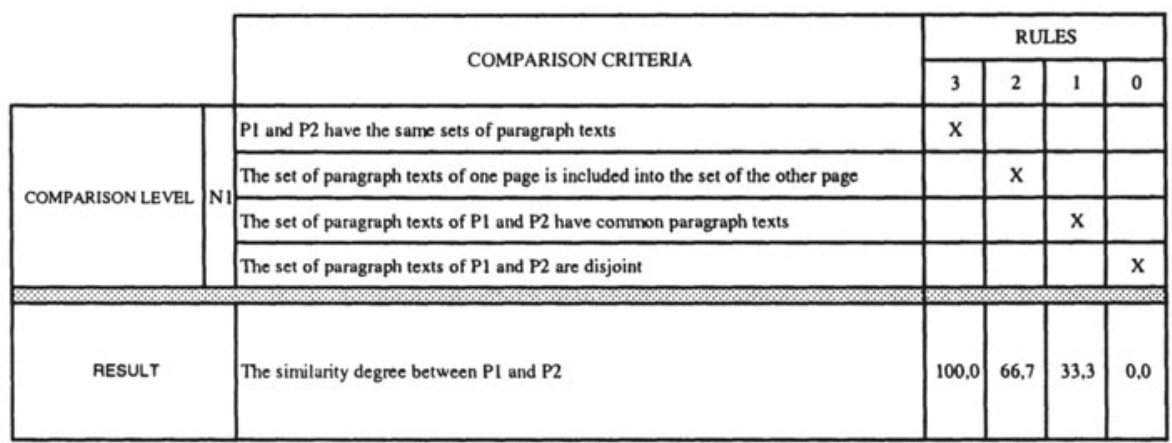

Table A. The rule set for structure type Text 


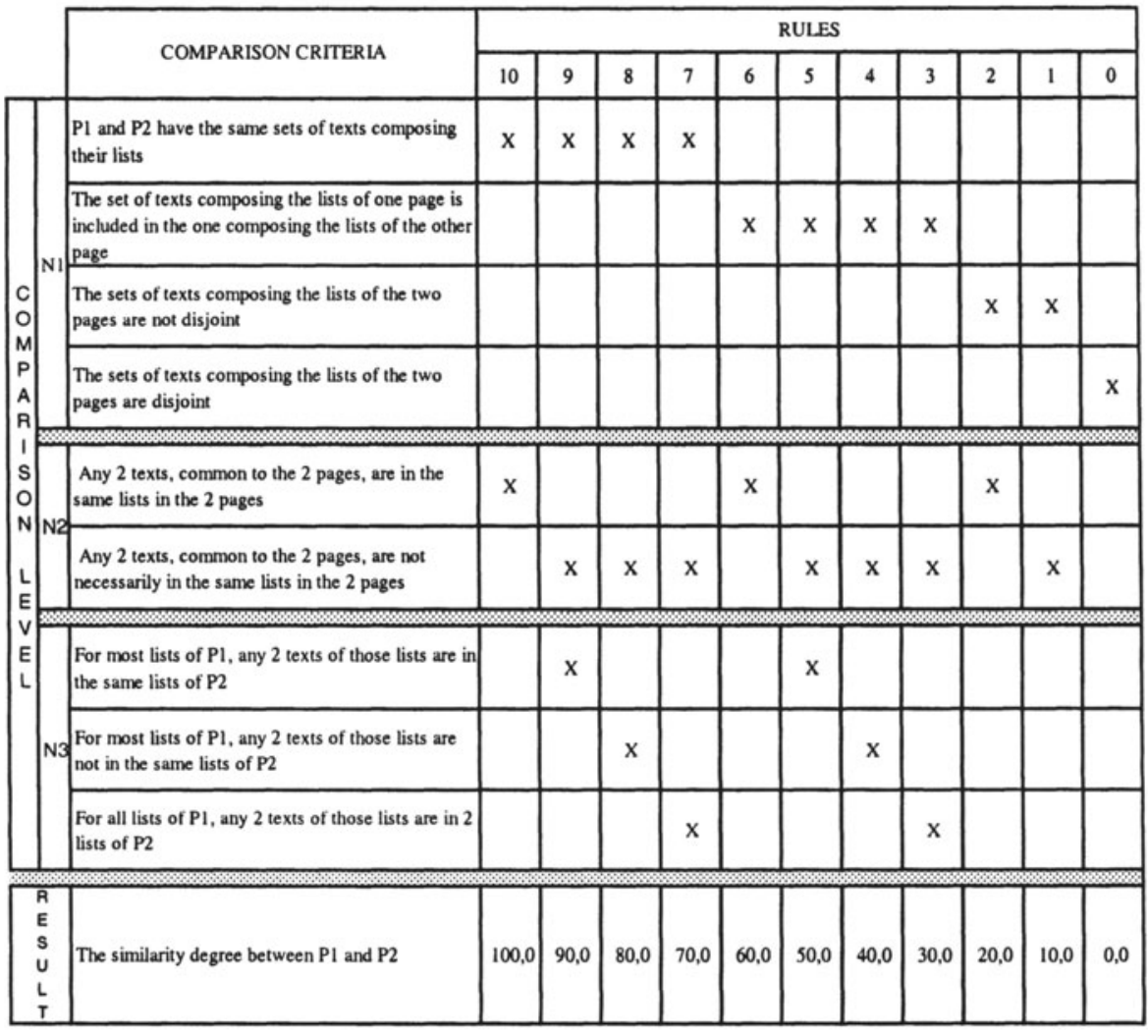

Table $B$. The rule set for structure type List 


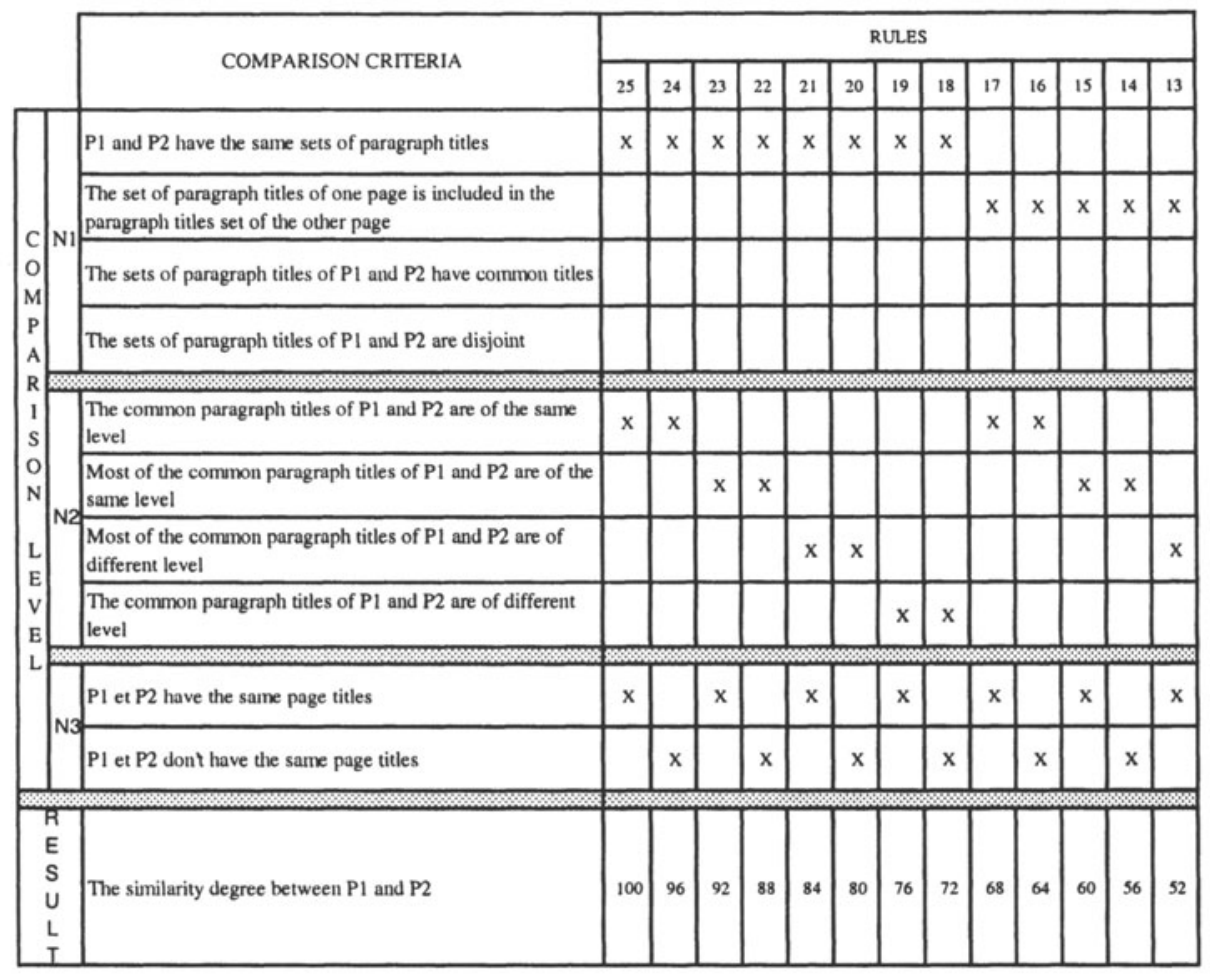

Table Cl. The rule set for structure type Title 


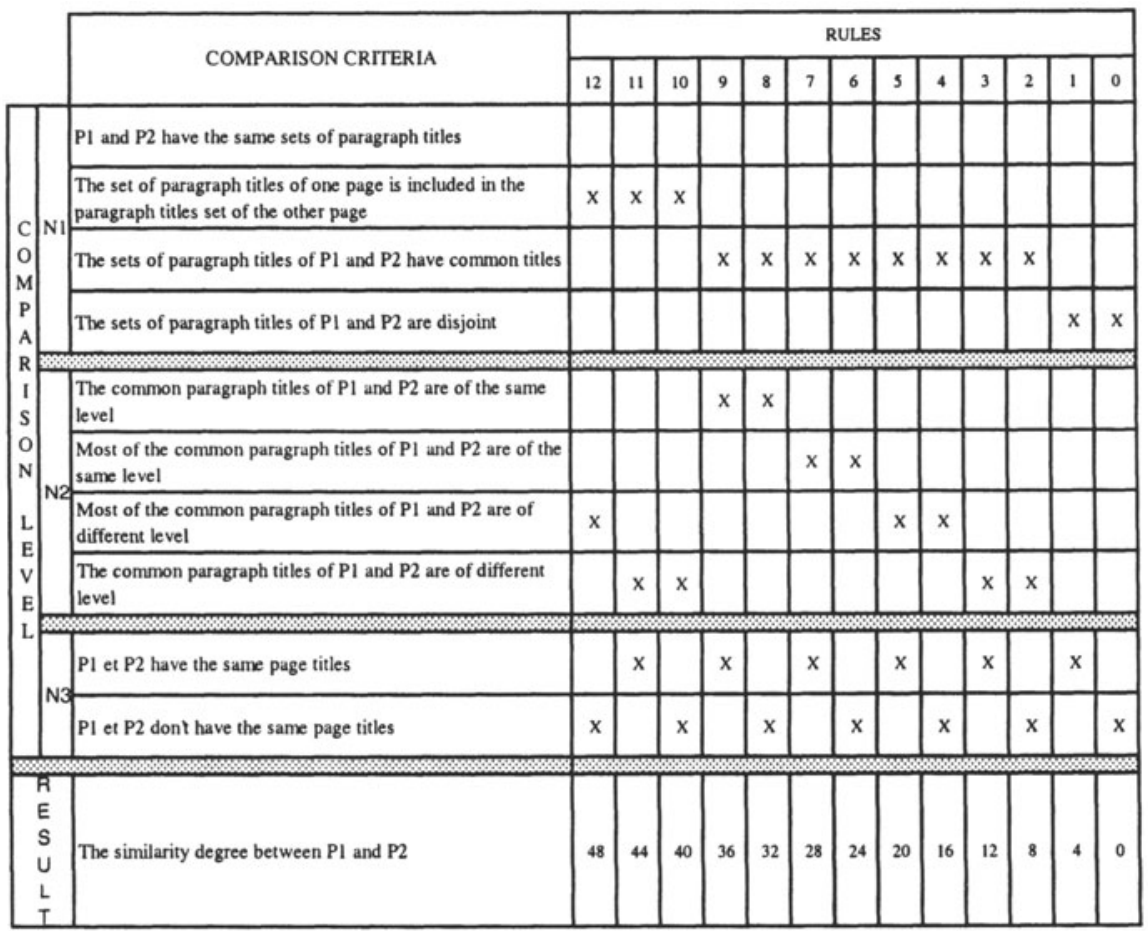

Table C2. The rule set for structure type Title (continued)

The result of this step is stored in $n$ matrices $S_{1}, S_{2}, . ., S_{n}$, one for each structure type. The component $(i, j)$ of matrix $\mathrm{Sk}$ is the degree of similarity between the $i$-th page of first web site and the $j$-th page of second web site according to the k-th structure type.

Using this combination of matrices, we constitute a global matrix in which each component measures the global similarity of the i-th page of first web site and $j$-th page of second web site as a weighted mean of structure similarities. The weights are determined by the designer at the beginning of the process based on the two main factors:

- the confidence of the designer in the structure types. If he knows that the original web sites interest is mainly due images whose logical arrangement did not obey to clear rules, he will assign a low value to title weight and a high value to image weight.

- the context of integration. If the web sites to be integrated describe similar organizations that have to be merged, the title weight must 
be high to give importance to the global structure and to maximize the consistency of the resulting web site structure.

\subsection{Merging step}

The comparison step leads to the elicitation of similarities between the web pages. The merging step will be guided by these similarities. Low values will be ignored. High values will lead to the merging of corresponding structures and pages. More precisely, the merging step is divided into two phases. The first phase is a pre-merging one. It aims at deciding the merging to be performed. The result of this first phase is a set of web pages pairs to be merged. The second phase is a unification process based on this set. The result is an instance of the conceptual schema described above (Figure 2).

\subsubsection{Pre-fusion step}

The merging of web pages is based on the global similarity matrix. Page $P_{1, i}$ (i-th page of the first site) can be merged with Page $P_{2, j}$ (j-th page of the second site) if the global similarity between the two pages is superior to a given threshold. This threshold may be fixed by the designer at the beginning of the integration process. Another possible way is to define a threshold for each structure type. This second possibility allows the designer to control more precisely the comparison criteria. These two possibilities lead to two integration modes: a standard mode in which a global threshold is used, and a customized mode in which the merging is based both on a global threshold and on structure thresholds. Let's consider a pair of pages (P,P') characterized by a global similarity of $40 \%$. These similarities are deduced from a $60 \%$ title similarity and a $0 \%$ image similarity. A global threshold of $20 \%$ will conclude to the merging of $\mathrm{P}$ and $\mathrm{P}$ '. However, if an image threshold of $10 \%$ is required, $\mathrm{P}$ and $\mathrm{P}$ ' are not merged.

In the worst case, the pre-merging step may lead to the following conclusion: no merging is possible. In all other cases, two situations may occur:

Case 1. Several pages may be merged into a unique page.

Case 2. Several pages may be merged with several pages in the other web site. 
Although the first case is not trivial, the second one is much more complicated. It requires a decision concerning the mergers to perform. We defined several merging scenarios. The choice of a scenario will depend on the integration strategy. For example, suppose the comparison of three pages in the first site with four pages in the second site leads to the following global similarity matrix:

\begin{tabular}{|l|l|l|l|l|}
\cline { 2 - 5 } \multicolumn{1}{c|}{} & P21 & P22 & P23 & P24 \\
\hline P11 & $60 \%$ & $40 \%$ & $00 \%$ & $00 \%$ \\
\hline P12 & $60 \%$ & $30 \%$ & $60 \%$ & $00 \%$ \\
\hline P13 & $50 \%$ & $00 \%$ & $00 \%$ & $00 \%$ \\
\hline
\end{tabular}

If the global threshold is equal to $10 \%$ and if the designer chose a standard integration, we have six different possible mergers. They are represented in the following bipartite graph in which the arcs are labelled with global similarities between web pages (Figure 3).

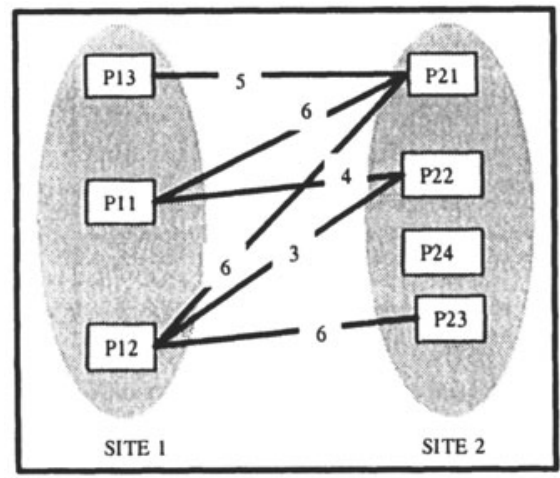

Figure 3. Example of similarity calculus

Depending on the integration context, the decision in such a situation may be different. For example, let's suppose that the objective of the integration is to include the web site S1 in the web site S2. For example, web site 2 is the product catalog of a company and web site 1 is a general documentary database about the products. The objective of the integration is to enrich the web site $S 2$ by all the $S 1$ relevant pages. In such a case, if a page $P$ belonging to $S 1$ is similar to several S2 web pages, it will be merged with the most similar page of S2. In this case, the set of mergers is limited to those mentioned in Figure 4b. This integration context can be compared to the strategy of 
preferred view proposed by Navathe to deal with conflicts between schemas to be integrated [3].

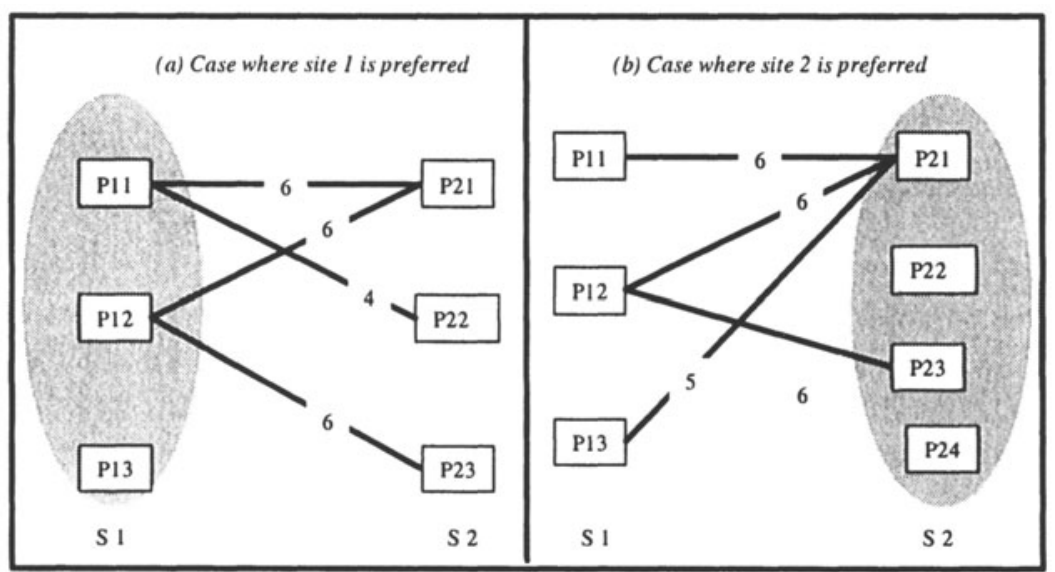

Figure 4. Cases where a site is preferred

To avoid redundancies in the resulting site, a number of merge-able pages must be discarded. In Figure 3, P21 is equally similar to P11 and P12. We can't merge both P21 with P11 and with P12. The solution is to provide the designer with the two scenarios described at Figure 5.

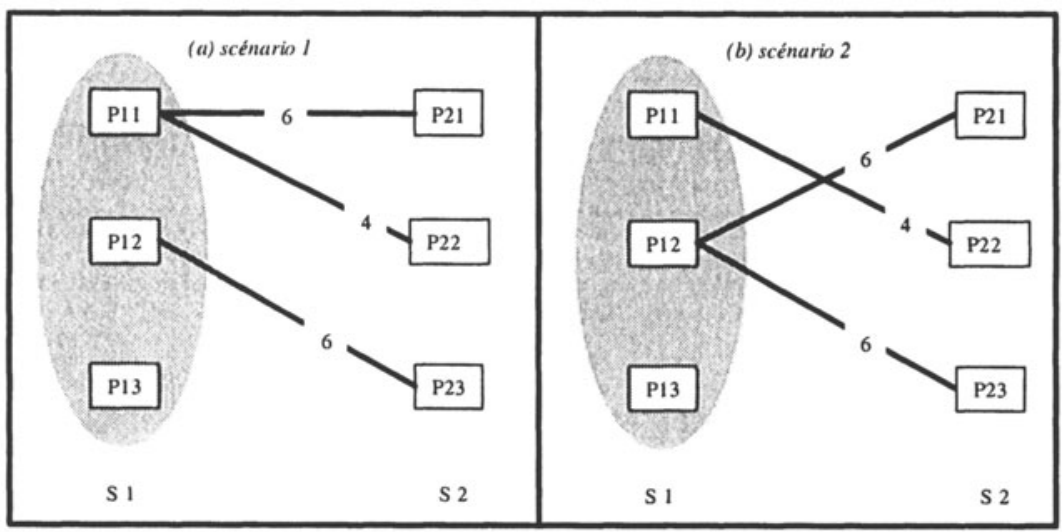

Figure 5. Two integration scenarios 
If the designer opts for scenario 1 , the resulting site will be composed of three pages:

- the result of P21 and P22 integrated with P11,

- the result of P23 and P12 integration,

- and page P13.

In brief, in such a context, the merging algorithm is the one sketched at Figure 6.

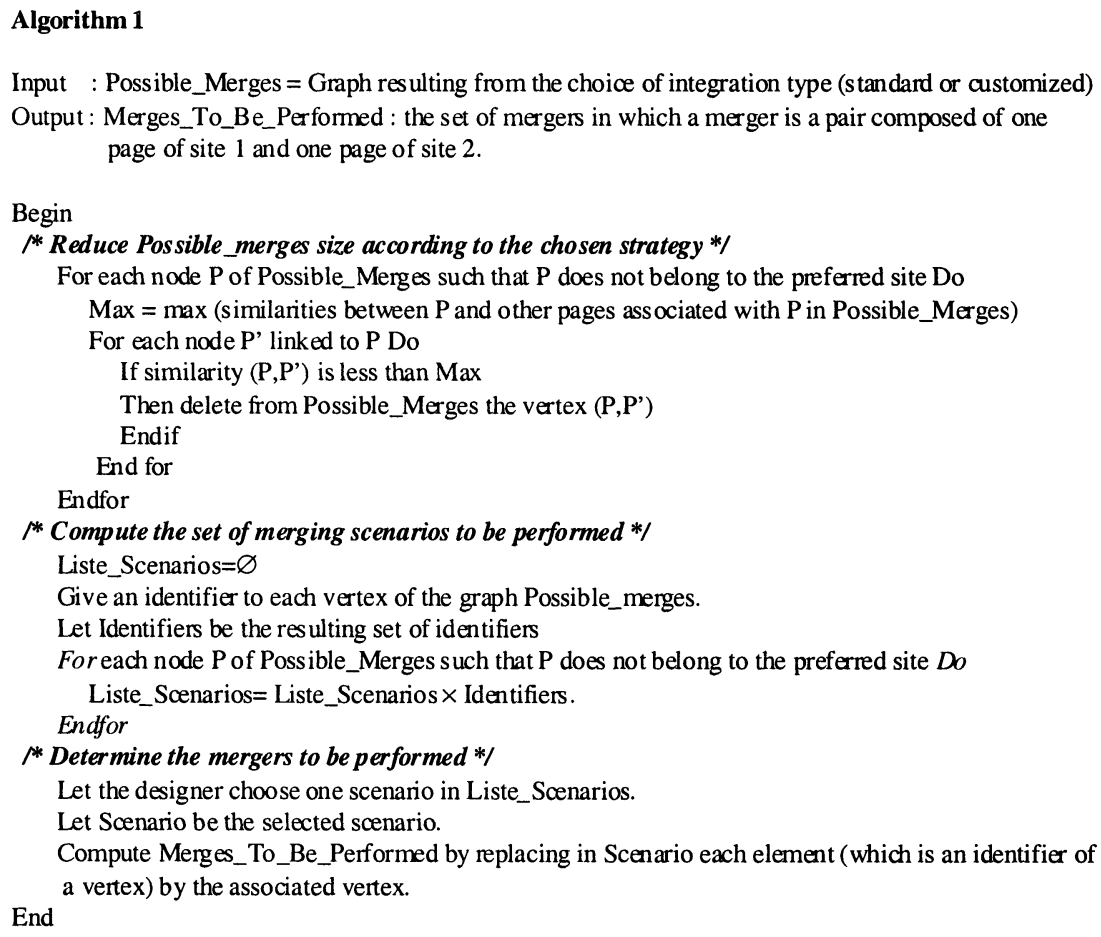

\section{Figure 6. First merging algorithm}

Let's consider the case where the integrator does not have a preferred site. For example, a company wants to merge two business units and considers their two web sites as equivalent. In such a case, the set Merges_To_Be_Performed is not transformed. On the contrary, in the case of a preferred site, in order to avoid redundancies, mergers have to be sorted. The order criterion is based on similarity degrees. First most similar pages are merged. The process ends with the unification 
of lower similarity pages. At each step of merging, a given page cannot be unified if it has already be merged with another page. In the example sketched at Figure 1, the first and unique unification will deal with P11, P12, P21 and P22 merging.

In the case where there is no preferred site, the merging choice algorithm is given at Figure 7 :

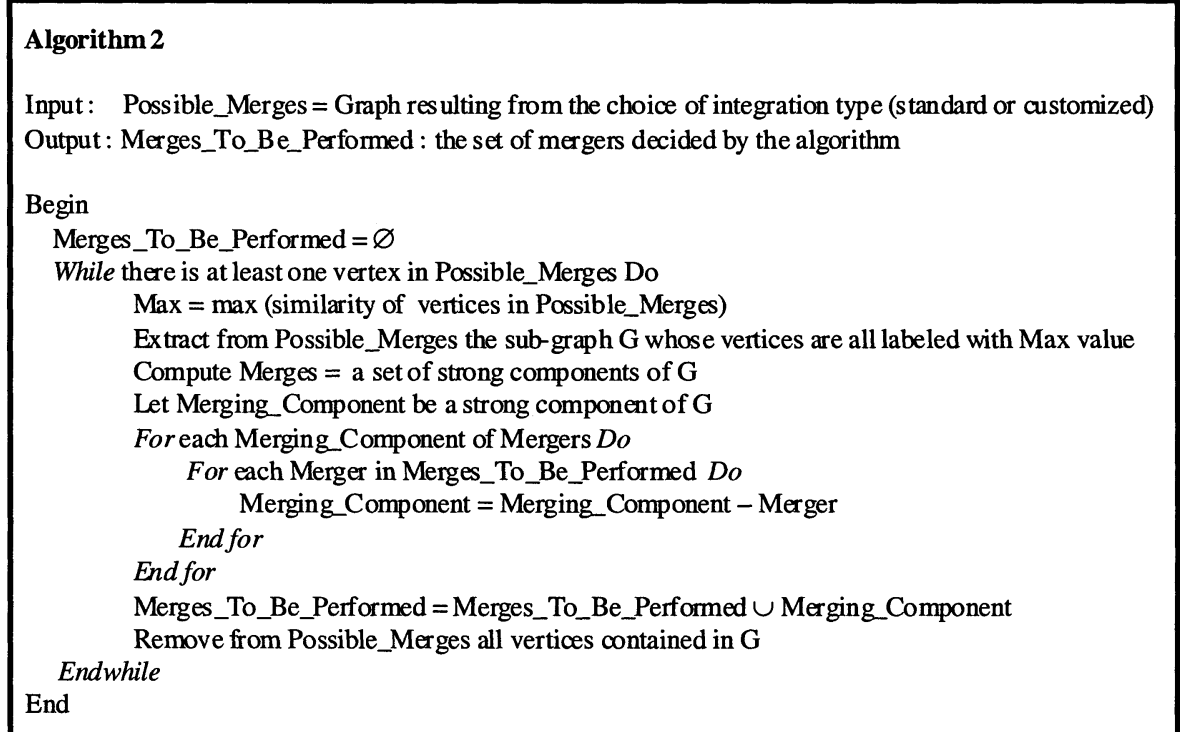

Figure 7. Second merging algorithm

In the pre-merging description, we have sketched the case of two integration strategies (standard vs. customized) to be combined with two integration situations (case where a site is preferred and case where the two sites are equally considered). Other integration strategies and situations require adaptations of previous algorithms.

\subsubsection{The integration or merging}

This phase starts with the result of the previous pre-merging algorithm. It also depends on the integration strategy and the integration situation (or context) which allow the designer or the CASE tool to define the resulting instance of the conceptual schema 
representing the integrated web site. If we are in the context of a preferred site, this instance is the preferred web site instance enriched with merge-able pages of the other web site. The graph representing the navigational model of the web site is also based on the original preferred web site graph. However, several links are added to this graph.

Suppose that we integrate sites $S 1$ and $S 2$ and that $S 1$ is the preferred site. Suppose that $\mathrm{P} 2 \mathrm{i}$ is a page from $\mathrm{S} 2$ that must be merged with a page P1i from S1. At least five different cases may occur (Figure 8):

- Case (a): P2i contains an intra-site link towards $P 2 \mathrm{j}$. $P 2 \mathrm{j}$ is also a page of $S 2$ and $P 2 j$ has no correspondent in $S 1$, that means $P 2 j$ won't undergo any merge with a page from $S 1$.

- Case (b): P2i contains an intra-site link towards P2j. P2j is also a page of $S 2$, which is to be merged with the page $P 1 j$ from $S 1$.

- Case (c): P2i contains an extra-site link towards a page not located in $\mathrm{S} 1$.

- Case (d): P2i contains an extra-site link towards a page P1j from S1, different from P1i.

- Case (e): There is an extra-site link from P2i to P1i and the pair (P1i, P2i) must be merged. 


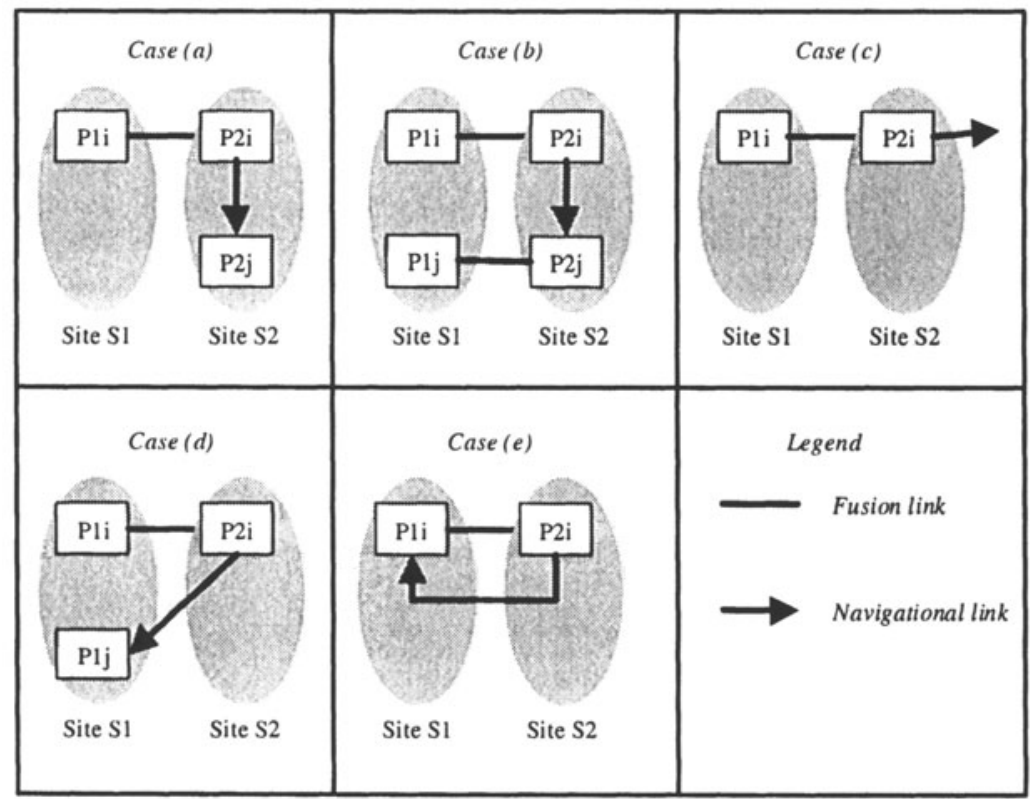

Figure 8. Interaction between mergings and hypertext links

For each case, an integration is proposed at Figure 9. For example, in case (a), the intra-site link from $P 2 i$ to $P 2 j$ is added to the set of links issued from $\mathrm{Pli}$ and becomes an extra-site link.

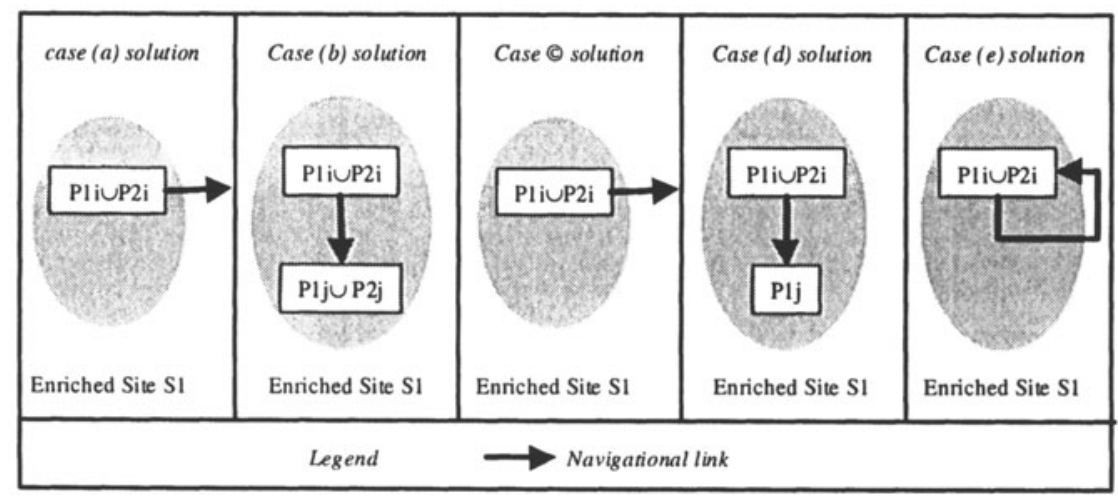

Figure 9. Integration with preferred site S1 
In the case where the integration does not privilege one site, the conceptual schema instance for the resulting site is the union of original site instances, except the hypertext links. The graph of the resulting site is elaborated by defining a solution for each case of Figure 8. The solutions are describes at Figure 10. For example, if no site is preferred and if we are in case (a), an intra-site link from the page resulting from the union of $P 1 i$ and $P 2 i$ to the page $P 2 j$ is generated.

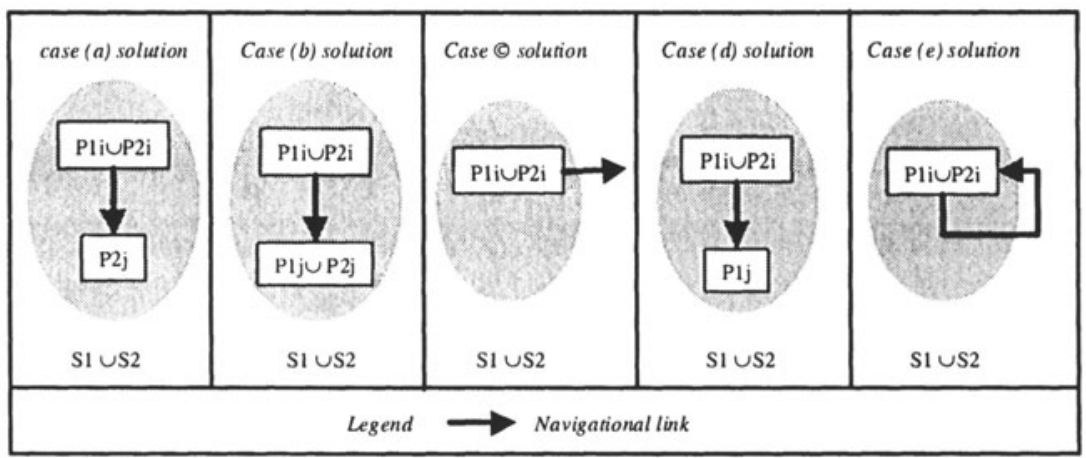

Figure 10. Integration without preferred site

Moreover, if the welcome pages of both sites were not sufficiently similar to be merged, a new welcome page is added to the resulting site. Its structure is limited to a page title "Welcome page" and to two intra-site links directed towards the two original welcome pages.

\section{$2.4 \quad$ Restructuring step}

This step must be conducted by the designer. Its objective is to improve the resulting web site. It consists in scanning the resulting web site. This analysis may lead to several transformations. For example:

- the navigation through the web site may reveal inconsistencies, for example a bad title hierarchical structure,

- cyclic graphs in a web site are obviously authorized. However, small cycles are to be avoided,

- if the designer detects a page containing redundant images (different sizes, different colors, different viewpoints but too many 
similarities), he will generally eliminate one of the two redundant images.

Our concern here is only about the content of the resulting web site. The definition of layout and aesthetic features can be delegated to a webmaster.

We have described in this section the main concepts of our web site integration approach. The next section is an illustration based on a real-life example.

\section{ILLUSTRATION}

This case study is related to the integration of two real-life web sites. However, we have substituted the names and largely simplified the contents. The first site (Site 1) is associated with a private training science institute located in Champagne-Ardennes. The second site (Site 2) describes a similar institute dedicated to computer science training located in Bordeaux. We suppose that these two institutes merge. Our aim is to perform the integration of their web sites. The navigational models of these two sites are presented at Figure 11.

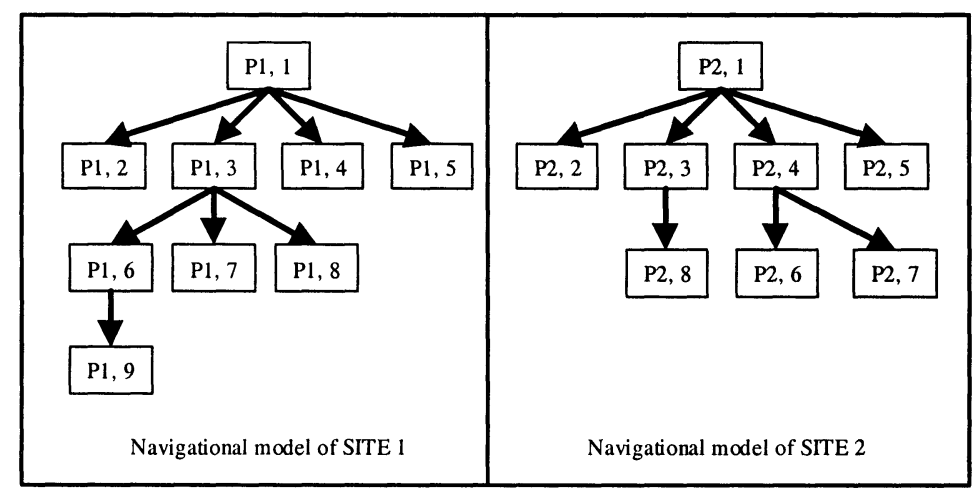

Figure 11. Navigational models of the two sites

The two sites pages are described in Annex B according to the grammar given in Annex A.The integration first requires the calculus of elementary similarities between comparable pages. The result of the 
comparison of SITE1 pages with SITE2 pages, is given below, structure type by structure type:

1. Regarding image, table and form structures, every page of SITE2 is $0 \%$ similar to every page of SITE1

2. Regarding the text structure:

- pages P2,6 and P2,7 of SITE2 are $67 \%$ similar to page P1,8 of SITE1,

- page P2,8 of SITE2 is $34 \%$ similar to page P1,9 of SITE1.

3. Regarding the title structure:

- page P2,3 of SITE2 is $4 \%$ similar to page P1,9 of SITE1,

- page P2,4 of SITE2 is $96 \%$ similar to page P1,8 of SITE1

- page P2,8 of SITE2 is $40 \%$ similar to page P1,9 of SITE1.

4. Regarding the list structure:

- page P2,3 of SITE2 is $10 \%$ similar to page P1,6 of SITE1,

- page P2,4 of SITE2 is $100 \%$ similar to page P1,8 of SITE1.

To define the global similarity between pages of the two sites, we use the following weights:

- 4 for the text structure,

- 3 for the title structure,

- 2 for the list structure,

- 1 for the form structure,

- 0 for the table, form and picture structures.

Our choice is motivated by the fact that educational sites use extensively text information but give little importance to tables and pictures.

A standard integration using the weights and the structure similarities leads to the possible mergers represented at Figure 12. 


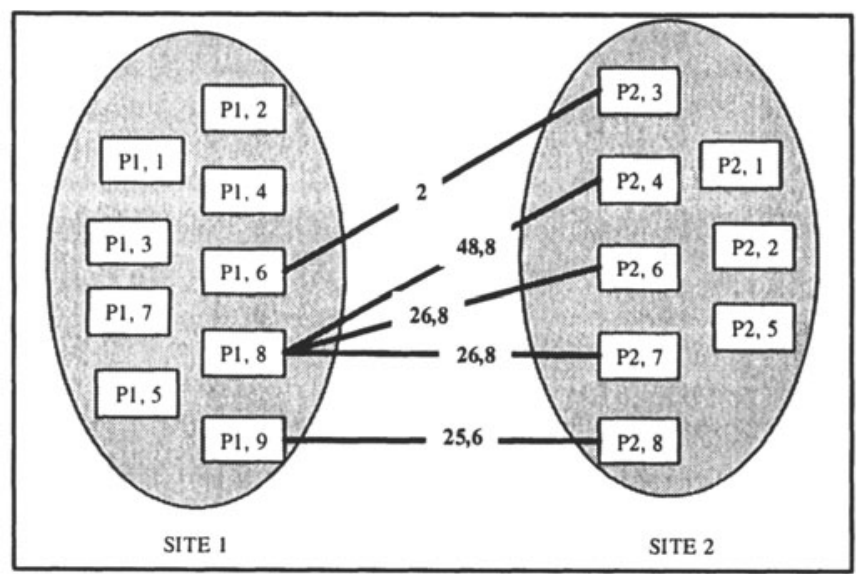

Figure 12 Graph of possible merges

We have supposed that the global similarity threshold is equal to $1 \%$. Let's consider successively three different integration contexts:

Context 1: SITE1 is preferred to SITE2. In this case:

- all the pages of SITE1 will be maintained in the resulting web site,

- P1,6 will be enriched by page P2,3,

- P1,8 will be enriched by the pages $\mathrm{P} 2,4, \mathrm{P} 2,6$ and P2,7

Context 2: SITE2 is preferred to SITE1. In this case:

- all the pages of SITE2 will be maintained in the resulting web site,

- P2,4 will be enriched by page P1,8

- P2,8 will be enriched by page P1,9

- P2,3 will be enriched by page P1,6

Context 3: None of the two sites is preferred. In this case:

- P2,4 will be merged with P1,8

- P2,8 will be merged with P1,9

- P2,3 will be merged with P1,6

- the remaining pages of SITE1 and SITE2 will be maintained in the resulting web site

- a welcome page is added to the resulting web site.

In each context, the corresponding navigational model is generated. 


\section{RELATED WORKS}

To the best of our knowledge, there is no literature about web site integration. However several research themes deal with related problems. First, view integration methodologies can be considered as a good framework to help in structuring a web site integration approach. Second, we summarize the state-of-the-art concerning web site conceptual modelling even if not all web site conceptual schemas can be used to compare and merge web sites. Ontology restructuring aims at building a unique ontology starting from different ones. In the same way, there is an analogy between an ontology structure and a web site organization. This analogy can be used to improve our web site integration approach. Let us mention works about Enterprise Applications Integration. However, this field is concerned with practical interfacing of applications. We claim that a semantic integration leads to higher quality web sites.

View integration methodologies aim at defining a sequence of steps leading from two or more conceptual schemas to a unique one representing the whole structure of data represented in the initial schemas $[3,8,9,10,14,15,20]$. It is particularly useful when organizations hold data in a variety of database systems. In all proposed approaches, the main problem mentioned remains the schema comparison, that is how can we make more automatic as well as more semantic the confrontation of objects in order to establish their similarities [18]. Web sites represented with conceptual schemas can be integrated if main semantic information about web site content and structure is captured by these schemas.

As pointed out by [2], it is probably the case that, in a few years, all information systems will be web-based. This new generation of web information systems requires to adapt conceptual modeling approaches. There is a need for web site conceptual modeling. [6] described a conceptual modeling approach to extracting and structuring data from the web. They proposed a conceptual model instance providing the relationships among the objects of interest, the cardinality constraints for these relationships, a description of the possible strings that can populate various sets of objects, and possible context keywords. Like a number of data models that have been developed during the last years, this approach aims at extracting data from the web [7]. They focus on inter-document structures, with little 
attention to intra-document structures. As a consequence, they represent the web data at a very low level. [13] proposed a conceptual for HTML that is able to represent any complex hierarchical structure within the web document at a high level that is close to human conceptualization of the documents. They also described how to convert HTML documents based on this conceptual model. [1] uses the UML notation to describe the content and the structure of XML documents. A meta-model is proposed. Each instance is a schema of classes representing an XML document. A class in the schema can either represent objects described in the document or contain some information, called meta-data, associated with the description of the type of structures (images, videos, texts, etc.) used in the document. However these conceptual models are not convenient for our integration problem.

Central to the web documents is the concept of an ontology. The latter codifies a shared and common understanding of some domain. An ontology specifies the fundamental objects and relations among them. As a consequence, ontology restructuring can be useful for the integration process. [16] have outlined a scalable framework for a system that enables interoperation between knowledge sources to answer user queries. In their paper, [11] described a method supporting semi-automatic ontology acquisition from corporate Intranet of a company. A method to build domain ontologies from texts is presented by [17]. [5] discussed how hierarchical clustering algorithms can be employed to automatically construct basic ontologies. [21] reviewed the use of ontologies for the integration of heterogeneous information sources. They showed how to use ontologies for the identification and association of semantically corresponding information concepts. Finally, [4] presented a general framework for ontology integration within semantic web applications.

\section{CONCLUSION AND FUTURE RESEARCH}

In this paper, we presented an approach for integration web sites which can be seen as a part of an integration methodology for ontologies. We concentrate on the situation that arises when a company is facing reorganization of its business units or is involved in merger or acquisition operation. In such a situation, the company must 
show an ability to rapidly develop a new web site integrating the main features of the existing web sites. The integration process follows a top-down approach based on a Entity-Relationship conceptual representation of each existing web site. A four-step integration process is applied. The emphasis is put on the comparison and merging steps for which relevant heuristics are proposed. Our objective is to minimize the designer's effort by automating as much as possible these two main phases.

Our approach tackles for the first time the difficult problem of web site integration. It also proposes a formal and automatic integration process. It ensures that most of the treatments involved are automated. It allows the designer to reuse existing web site and perform rapidly an integration process.

We described a first cut methodology which needs some refinements, in particular for the measures. This refinement will be conducted by an extensive experimentation of the proposed approach on several real-life web sites, thus allowing us to evaluate the quality of the approach. This paper concentrates on HTML document integration. Another further research is to generalize the approach to any document type (eg. XML) including comparison of heterogeneous web sites. Tools performing texts and images analysis are needed. Finally, integrating these tools within a framework of an appropriate ontology will facilitate the semi-automatic integration process.

\section{REFERENCES}

[1] Amous I., Chrisment $C$ and Sèdes F. Etendre une Approche Méthodologique pour la Réingénierie des Sites Web, Revue ISI, Interopérabilité des SI, 6/2001, pp 119144.

[2] Atzeni P., Databases and the World Wide Web, LNCS 1725, SOFSEM'99, Theory and Practice of Information.

[3] Batini, C., Lenzerini, M., Navathe, S. A Comparative Analysis of Methodologies for Database Schema Integration, ACM Computing Surveys, 18(4), Dec, 1998.

[4] Calvanese D., de Giacomo G., Lenzerini M., A Framework for Ontology Integration, Semantic Web Working Symposium, 30 July-1 August 2001, California, USA.

[5] Clerkin P., Cummingham P., Hayes C., Ontology Discovery for the Semantic Web Using Hierarchical Clustering, ECML/PKDD 2001, Workshop on Semantic Web Mining, Fribourg, 2001. 
[6] Embley D, Campbell D.M, Jiang Y.S, Liddle S.W., Ng Y.K, Quass D.W, Smith R.D., A Conceptual Modeling Approach to Extracting Data from the Web, Proceedings of ER'98, Singapore.

[7] Florescu D., Levy A., Mendelzon A., Database Techniques for the World Wide Web: A survey, SIGMOD Record, 27(3), 1998.

[8] Frank, H., Eder, J., Towards an Automatic Integration of Statecharts, Proceedings of ER'99, LNCS 1728, Paris, 1999.

[9] Gotthard, W., Lockemann, P.C., Neufeld, A., System-guided View Integration for Object-Oriented Databases, IEEE Trans. Knowledge Data Engineering, February, 1992.

[10] Kashyap, V., Sheth, A., Semantic and schematic similarities between database objects: a context-based approach. VLDB Journal, 5(4), 1996.

[11] Kietz, J.K, Maedche A., Voltz R., A Method for Semi-Automatic Ontology Acquisition from a Corporate Intranet, EKAW'2000, Workshop on Ontologies and Texts, Juan Les Pins, 2000.

[12] Lammari N., An Algorithm to Extract IS-A Inheritance Hierarchies from a Relational Database, Proceedings of ER'99, LNCS 1728, Paris, 1999.

[13] Liu, M., Ling, T.W., A Conceptual Model for the Web, ER'2000, Salt Lake City.

[14] McBrien, P., Poulovassilis, A., A Formalisation of Semantic Schema Integration, Information Systems, 23(5), 1998.

[15] Métais, E., Kedad, Z., Comyn-Wattiau, I., Bouzeghoub, M., Using Linguistic Knowledge in View Integration: Toward a Third Generation of Tools, Data and Knowledge Engineering, 23(1), 1997.

[16] Mitra P. Wiederhold G., Kersten M., A Graph-Oriented Model for Articulation of Ontology Interdependencies, EDBT2000, LNCS 1777, Springer Verlag, 2000.

[17] Nobecourt J., A Method to Build Formal Ontologies from Texts, EKAW'2000, Workshop on Ontologies and Texts, Juan Les Pins, 2000.

[18] Parent, C., Spaccapietra, S., Issues and Approaches of Database Integration, Communications of the ACM, 41(5), 1998.

[19] Quass D.W, Smith R.D., A Conceptual Modeling Approach to Extracting Data from the Web, Proceedings of ER'98, Singapore.

[20] Thieme, C., Siebes, A., Guiding Schema Integration by Behavioural Information, Information Systems, 20(4), 1995.

[21] Wache H., Vögele T., Visser U., Stuckenschmidt H. Schuster G., Neumann H., Hübner S., Ontology-Based Integration of Information - A Survey of Existing Approaches, IJCAI'2001, Ontology and Information, 2001. 


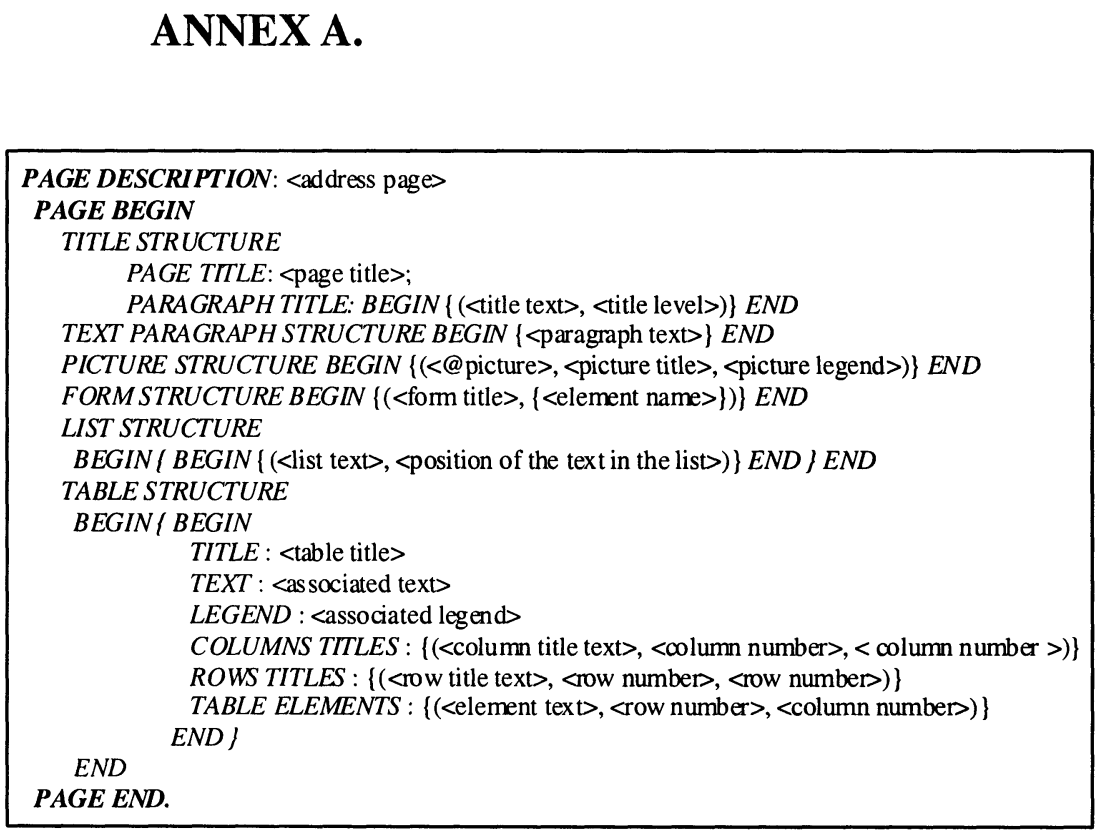

Figure 13. The grammar used to describe site pages 


\section{ANNEX B.}

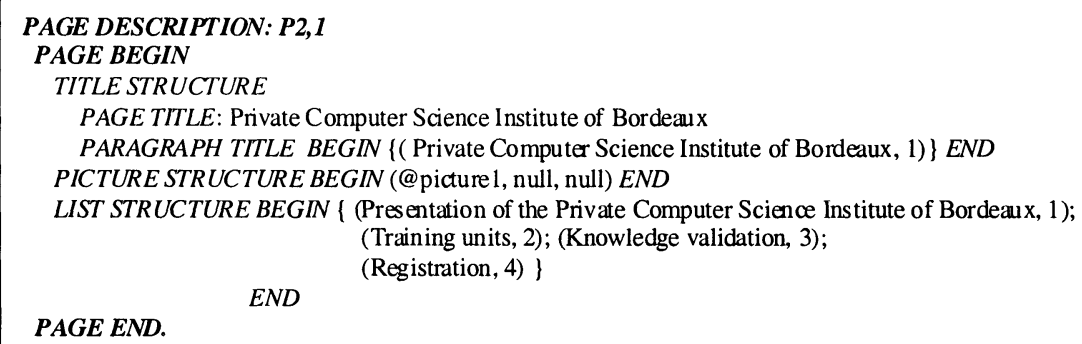

\section{PAGE DESCRIPTION: $\mathrm{P} 2,2$}

PAGE BEGIN

TITLE STRUCTURE

PAGE TITLE: Registration;

PARAGRAPH TITLE BEGIN \{(How to register, 1 ),

(Trainings are opened tOctober $3^{\text {rd }} 2001$ for a week, 2),

(Relevant registration documents, 2), (Registration fees, 2)\}

$E N D$

PICTURE STRUCTURE BEGIN \{(@picture1,null, null,),(@picture2,null, pcsi@bordeaux.fr) END LIST STRUCTURE BEGIN

$\{$ (identity document, 1), (diploma, 2), (employer certificate, 3)\}

\{(Individual fees : $500 \mathrm{~F}, 1$ ), (registration by a employer, $1000 \mathrm{~F}$ ) \}

PAGE END END

PAGE DESCRIPTION: $\mathrm{P} 2,3$

PAGE BEGIN

TITLE STRUCTURE

PAGE TITLE: Training units

PARAGRAPH TITLE BEGIN \{(Training units, 1), (OptionTraining1 , 2), (OptionTraining2, 2)\} END LIST STRUCTURE

$B E G I N\{($ Unit 1, 1), (Unit2, 2), (Unit3, 3) $\} ;\{($ Unit4, 1), (Unit5, 2) $\}$ END

PAGE END.

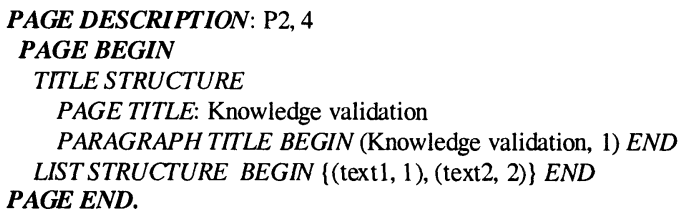

Figure 14. Description of the pages of SITE2 


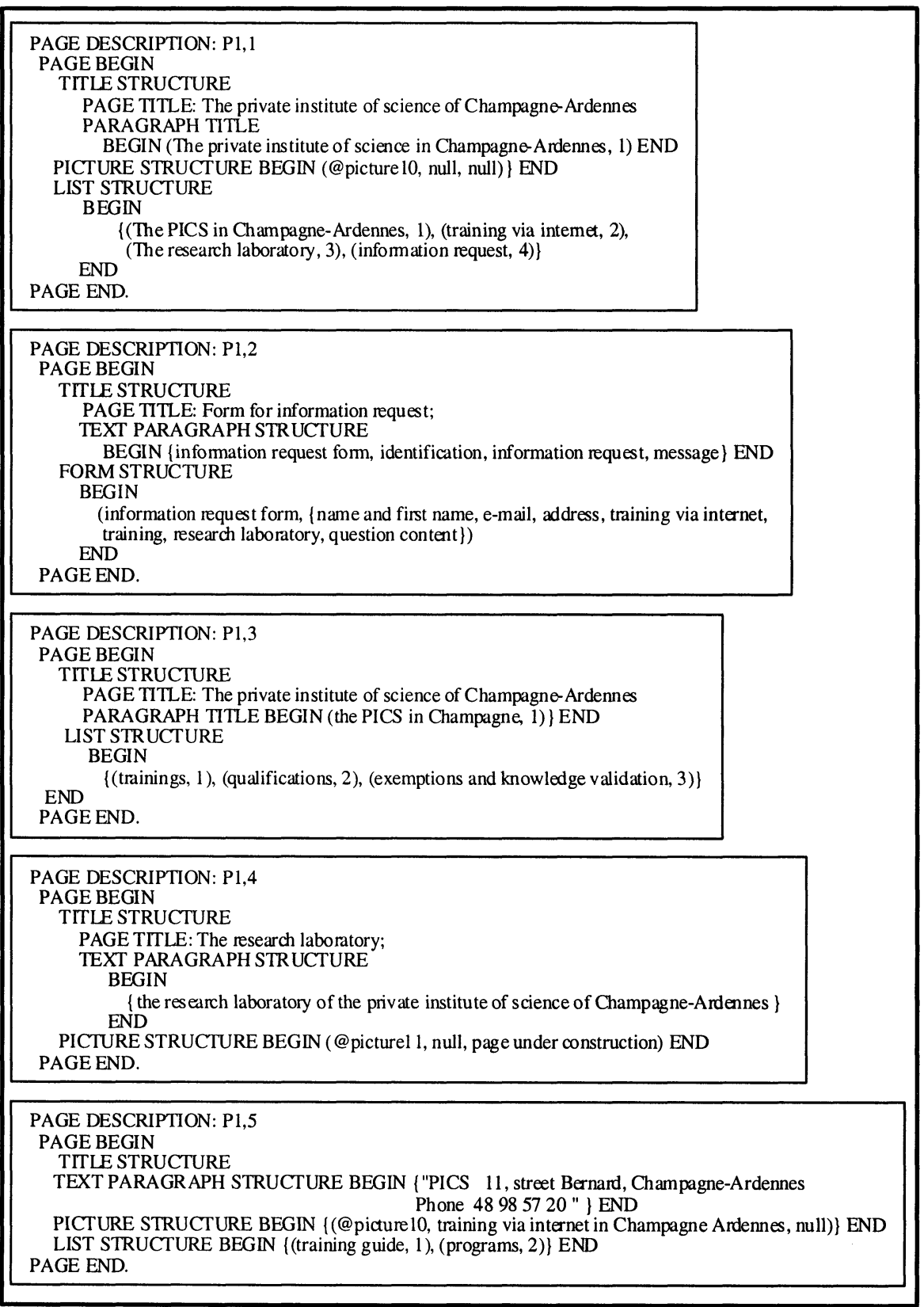

Figure 16. Description of the pages of SITE1 
